\title{
Ghost Haemoglobin Affecting the Efficacy of Phototherapy
}

\author{
Azizullah Langah1, Sara Sadiq2, Ali Akbar Siyal1 \\ ${ }^{1}$ Department of Pediatric Medicine, Peoples University of Health Sciences, Nawabshah, Pakistan \\ ${ }^{2}$ Department of Physiology, CMH Institute of Medical Sciences, Bahawalpur, Pakistan \\ Email: *dr.sarabhatti@gmail.com
}

How to cite this paper: Langah, A., Sadiq, S. and Siyal, A.A. (2019) Ghost Haemoglobin Affecting the Efficacy of Phototherapy. International Journal of Clinical Medicine, 10, 523-530.

https://doi.org/10.4236/ijcm.2019.1010042

Received: August 26, 2019

Accepted: October 12, 2019

Published: October 15, 2019

Copyright $\odot 2019$ by author(s) and Scientific Research Publishing Inc. This work is licensed under the Creative Commons Attribution International License (CC BY 4.0).

http://creativecommons.org/licenses/by/4.0/

\begin{abstract}
Introduction: Phototherapy is the treatment of choice for neonatal hyperbilirubinemia. It converts the unconjugated bilirubin from polar and neurotoxic Z-Z-bilirubin to the more polar photobilirubin. It has been hypothesized that high level of hemoglobin or hematocrit interferes in the effectiveness of phototherapy. The objective of the current study is to find out the association of hemoglobin/hematocrit to change in total serum bilirubin concentration during phototherapy. Methods: A prospective cohort study was conducted on 296 neonates with uncomplicated hyperbilirubinemia. Before initiating the phototherapy, hemoglobin, hematocrit and total serum bilirubin levels were measured. After treating the neonates with phototherapy using LED-light for 24 hours, the total serum bilirubin level was measured again. The data were analyzed by using SPSS version-20. Results: The majority of study participants were male $(60.8 \%)$, with mean postnatal age of $4.66 \pm 1.65$, having mean birth weight of $2.41 \pm 0.41$. Considering Pearson correlation, the hemoglobin had a significant inverse association with $\Delta \mathrm{TsB}_{0-24}$ while birth weight and postnatal age also had inverse association but $\mathrm{p}$-values were non-significant, while $\mathrm{TsB}_{0}$ had presented a very weak but significant positive association. The results might be evident of the fact that increase in hemoglobin concentration results in minimal change in total serum bilirubin concentration during phototherapy. Conclusion: Current study found a significant effect of hemoglobin on efficacy of phototherapy, as the change in total serum bilirubin concentration is inversely correlated with the level of hemoglobin. This is important for the physician in treating neonates with hyperbilirubinemia by using phototherapy.
\end{abstract}

\section{Keywords}

Hyperbilirubinemia, Neonatal Jaundice, Phototherapy 


\section{Introduction}

Neonatal Jaundice is not an uncommon condition, approximately $2 \%-6 \%$ of affected neonates require treatment [1]. Over the past few decades, phototherapy is the treatment of choice for neonatal hyperbilirubinemia, beside intravenous immune globulin (IVIG) and exchange transfusion. Phototherapy prevents the bilirubin to touch the extremely high levels in turn, preventing kernicterus (encephalopathy due to high levels of bilirubin) [2] [3].

The mechanism of action of phototherapy is that it converts the unconjugated bilirubin from polar and neurotoxic Z-Z-bilirubin to the more polar form known as photobilirubin. This transformed photobilirubin consists of configurational Z-E and E-Z-bilirubin isomers and structural E-Z and E-E-lumirubin isomers [3] [4] [5]. These transformed isomers are easy to excrete in both bile without undergoing conjugation and in urine as well [6]. The fact that the mechanism of phototherapy induced transformation occurs predominantly either intravascularly or extravascularly, is under a great debate but the majority favors the idea of intravascular phototherapy induced transformation [7].

In vitro number of studies reported that hemoglobin competes the bilirubin for absorbing light during phototherapy [7] [8] [9] as the erythrocytes do not have nuclei or other cellular organelles so act as the main light absorber [9] [10]. It has been hypothesized that high level of hemoglobin or hematocrit interfered in the effectiveness of phototherapy and only one of the studies confirmed it in vivo [1]. It is very important to confirm this hypothesis in our clinical setting so that one can understand the underlying basic mechanism going-on in phototherapy and to suggest a treatment strategy in neonatal hyperbilirubinemia. The objective of the current study is to find out the association of hemoglobin/hematocrit to change in total serum bilirubin concentration during phototherapy. The hypothesis is that the increase in hemoglobin concentration reduces the efficacy of phototherapy.

\section{Methods}

A prospective cohort study was done from August 2018 up to March 2019 in the neonatal intensive care unit of Peoples University of medical and health sciences (PUMHS) Nawabshah, Pakistan. OpenEpi calculator was used for calculating the sample size which was 306 while the sample population was selected using randomization. The inclusion criteria followed were: 1) healthy term or late preterm (more than 34 weeks of gestation) newborn having hyperbilirubinemia 2) no signs for hemolytic disease 3) clinical signs of jaundice 4) birth weight must be more than $1800 \mathrm{~g}$ 5) postnatal age should be more than $24 \mathrm{~h}$ but less than 7 days and 6) those who were not previously exposed to phototherapy. Those neonates were excluded from the study whose parents refuse to give the consent or who were either candidate for exchange transfusion or need double phototherapy or got Rhesus ( $\mathrm{Rh}$ ) isoimmunization or having a very high level or rapidly increasing level of total serum bilirubin concentration or patients diagnosed as a septic 
case. The basic demographic data including gestational age, postnatal age, gender, birth weight and type of feeding during phototherapy were recorded.

\section{Measurements.}

Before initiating the phototherapy, hemoglobin and hematocrit were measured. A trained phlebotomist collected blood samples from the vein/capillary by heel stick method. All the samples were transported under refrigerated temperature and centrifuged within 4-hours of collection. The hemoglobin and hematocrit levels were analyzed by using fully auto hematology analyzer (Model: HL-2400 Plus) in pathology laboratory of PUMHS Nawabshah.

Total serum bilirubin level was measured at the start $\left(\mathrm{TsB}_{0}\right)$ and after giving phototherapy for 24 hours $\left(\mathrm{TsB}_{24}\right)$ in the capillary blood, drawn simultaneously with blood for hemoglobin/hematocrit. It was analyzed by using clinical chemistry analyzer (VitalabSelectra E-series) in pathology laboratory of PUMHS Nawabshah.

\section{Phototherapy:}

The instrument used for phototherapy was Infant phototherapy lamp (Model: XHZ-90L, Ningbo David Medical Device CO.LTD), having blue LED-light of 430 - $490 \mathrm{~nm}$ spectrum. Before initiating the trial, device was calibrated from the service provider company. Light irradiance was measured by using a radiometer before initiating the trial and during the phototherapy after every eight hours i.e. three times within 24 hours. It was measured at the head, abdomen and knees of the exposed infants and the mean values were calculated.

The distance between the mattress and the light source of phototherapy lamp was about $30 \mathrm{~cm}$ and it was adjusted by using a wood template. The average distance from the lamp to the skin of the infant was $22.5 \mathrm{~cm}$. Phototherapy was given to all infants continuously for 24 hours except in between thirty minutes of feeding and nursing care after every three hours. All the participants were naked except the area of diapers and eye pads. Phototherapy was avoided if direct hyperbilirubinemia is more than $20 \%$ of $\mathrm{Ts}_{0}$. Very few of the study participants were excluded from the study because of developing complications like skin rash (erythematous macular rash/purpuric rash), loose stool, overheating and dehydration.

\section{Ethics:}

The study got approval from the ethical review committee of PUMHS. Written informed consent was taken from the parents/guardian of the infants before collecting the blood samples.

\section{Statistical Analysis.}

The data were analyzed by using Statistical Package for Social Sciences (SPSS) version-20. Mean with standard deviation and range was calculated for numerical data while frequency and percentages for qualitative data. Hemoglobin, hematocrit, $\mathrm{TsB}_{0}, \mathrm{TsB}_{24}$, and change in total serum bilirubin after 24 hours of phototherapy $\left(\Delta \mathrm{TsB}_{0-24}\right)$ are presented as mean values with range. The linear regression model and Pearson correlation coefficient of $\Delta \mathrm{TsB}_{0-24}$ with hemoglobin, hematocrit, $\mathrm{TsB}_{0}$, postnatal age and birth weight were calculated. The scatter plot 
was drawn to report the distribution of data and association of hemoglobin with $\Delta \mathrm{TsB}_{0-24}$. P-value less than 0.05 was considered as statistically significant.

\section{Result}

A total of about 306 participants were included in the study but after excluding, those neonates whose parents refuse to give consent or having unsatisfactory results or developed complications, about 296 participants were finally analyzed. Majority of study participants were male (60.8\%), with mean postnatal age of $4.66 \pm 1.65$, having mean birth weight of $2.41 \pm 0.41$. Looking over the gestational age, majority of the participants were born during $37^{\text {th }}$ week of their gestation as presented in Figure 1. Among the participants, very few were on breast-fed during phototherapy and the mean light irradiance measured was $71.8 \pm 11.45$. The basic characteristics including demographic variables and irradiance data of study participants are mentioned in Table 1.

At the time of diagnosis, the mean $\mathrm{TsB}_{0}$ was $16.2 \pm 6.92$ while mean values of hemoglobin and hematocrit were $16.59 \pm 2.75$ and $45.78 \pm 6.18$ respectively. After giving phototherapy for 24 hours, the mean $\mathrm{TsB}_{24}$ was $13.15 \pm 3.76$, so the $\Delta \mathrm{TsB}_{0-24}$ concentration during 24 hours of phototherapy was $5.21 \pm 3.05$ as mentioned in Table 2.

The linear regression model and Pearson correlation coefficient are calculated in Table 3. The regression analysis showed significant impact of hemoglobin, hematocrit, $\mathrm{TsB}_{0}$ on $\Delta \mathrm{TsB}_{0-24}$ while non-significant impact of postnatal age and birth weight on $\Delta \mathrm{TsB}_{0-24}$. Considering Pearson correlation, the hemoglobin had significant inverse association with $\Delta \mathrm{TsB}_{0-24}$ while birth weight and postnatal age also had inverse association but p-values were non-significant, while hematocrit and $\mathrm{Ts}_{0}$ had presented a very weak positive association. The distribution of data in scatter plot with regression line is presented in Figure 2. It displayed a very weak negative correlation which might be evident of the fact that increases in hemoglobin concentration resulting in minimal change in total serum bilirubin concentration during phototherapy.

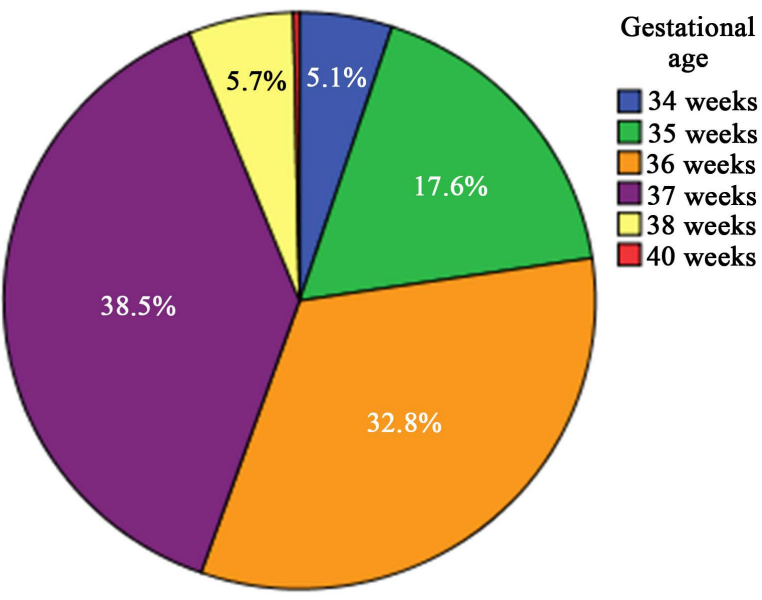

Figure 1. Gestational age of the study participants. 


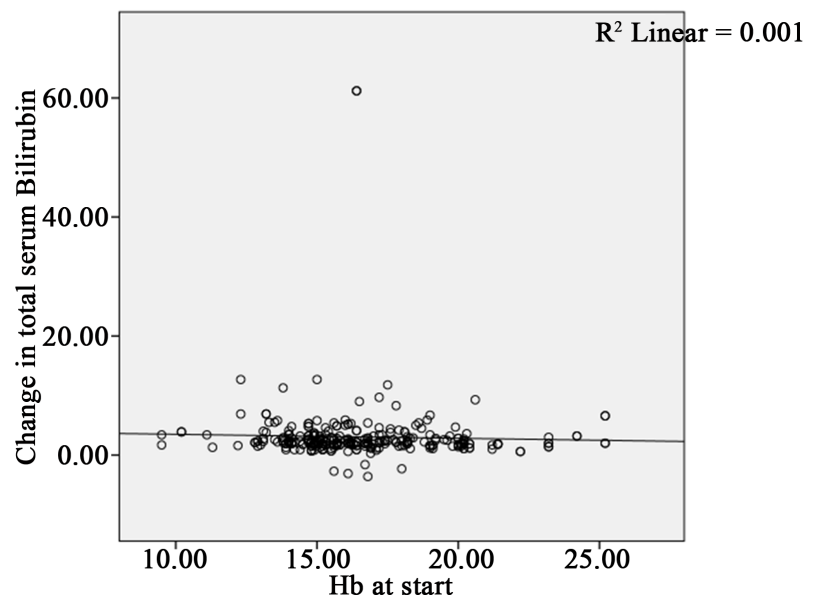

Figure 2. Scatter plot of change in total serum bilirubin during 24 hours of phototherapy versus hemoglobin concentration.

Table 1. Basic characteristics of study participants.

\begin{tabular}{|c|c|c|}
\hline \multicolumn{3}{|c|}{ Qualitative Variables } \\
\hline & $\mathrm{n}=296$ & $\%$ \\
\hline \multicolumn{3}{|l|}{ Gender } \\
\hline Male & 180 & 60.8 \\
\hline Female & 116 & 39.2 \\
\hline \multicolumn{3}{|l|}{ Type of feeding during phototherapy } \\
\hline Exclusive breast-fed & 81 & 27.4 \\
\hline Exclusive formula-fed & 131 & 44.3 \\
\hline Mixed breast-fed/ formula-fed & 84 & 28.4 \\
\hline \multicolumn{3}{|c|}{ Quantitative Variables } \\
\hline & Mean \pm SD & Range \\
\hline Postnatal age (days) & $4.66 \pm 1.65$ & $1.36-7.96$ \\
\hline Birth weight $(\mathrm{kg})$ & $2.41 \pm 0.41$ & $1.59-3.23$ \\
\hline Measured light irradiance $\left(\mu \mathrm{W} / \mathrm{cm}^{2} / \mathrm{nm}\right)$ & $71.8 \pm 11.45$ & $48.9-83.5$ \\
\hline
\end{tabular}

Table 2. Hemoglobin at the initiation of phototherapy and change in total serum bilirubin concentration during phototherapy.

\begin{tabular}{ccc}
\hline & Mean $\pm \mathrm{SD}$ & Range \\
\hline Hemoglobin $(\mathrm{g} / \mathrm{dl})$ & $16.59 \pm 2.75$ & $11.09-22.09$ \\
Hematocrit $(\%)$ & $45.78 \pm 6.18$ & $33.42-58.14$ \\
$\mathrm{TsB}_{0}(\mathrm{mg} / \mathrm{dl})$ & $16.2 \pm 6.92$ & $2.36-30.04$ \\
$\mathrm{TsB}_{24}(\mathrm{mg} / \mathrm{dl})$ & $13.15 \pm 3.76$ & $5.63-20.67$ \\
$\Delta \mathrm{TsB}_{0-24}(\mathrm{mg} / \mathrm{dl})$ & $5.21 \pm 3.05$ & - \\
\hline
\end{tabular}

$\mathrm{TsB}_{0}$, Total serum bilirubin concentration at the start of phototherapy; $\mathrm{TsB}_{24}$, Total serum bilirubin concentration after 24 hours of phototherapy; $\Delta \mathrm{TsB}_{0-24}$, change in total serum bilirubin concentration during initial 24 hours (difference between $\mathrm{TsB}_{0}$ and $\mathrm{TsB}_{24}$ ). 
Table 3. Linear regression analysis and pearson correlation.

\begin{tabular}{ccccc}
\hline & $\mathbf{R}$ & $\mathbf{R}^{2}$ & Coefficient $(95 \% \mathrm{CI})$ & $\mathrm{p}$-value \\
\hline Hemoglobin $(\mathrm{gm} / \mathrm{dl})$ & -0.035 & 0.001 & $-0.286-0.153$ & 0.005 \\
Hematocrit $(\%)$ & 0.055 & 0.003 & $-0.050-0.143$ & 0.023 \\
$\mathrm{TsB}_{0}(\mathrm{mg} / \mathrm{dl})$ & 0.054 & 0.003 & $-0.046-0.127$ & 0.035 \\
Postnatal age & -0.030 & 0.001 & $-0.458-0.268$ & 0.607 \\
Birth weight & -0.048 & 0.002 & $-2.043-0.838$ & 0.411 \\
\hline
\end{tabular}

$\mathrm{R}$, Pearson correlation; $\mathrm{R}^{2}$, regression; $\mathrm{CI}$, confidence interval; $\mathrm{TsB}_{0}$, Total serum bilirubin concentration at the start of phototherapy.

\section{Discussion}

The results of current study showed a weak negative association of hemoglobin with $\mathrm{TsB}_{0-24}$ with significant $\mathrm{p}$-value. To the best of our knowledge only one clinical study done in vivo reported hemoglobin as a competitor of bilirubin during phototherapy [1]. On the other hand Granati et al. did in vitro study and reported significant increase in degradation of bilirubin as the hematocrit level declined during phototherapy but he did not find any of such association in vivo [11]. The fact that hemoglobin compete with bilirubin to absorb phototherapy light was confirmed in vitro by Lamola et al., he used a semi-empirical skin model which showed the competition of hemoglobin with bilirubin and this increased if wavelength increased $400 \mathrm{~nm}$ to $460 \mathrm{~nm}$ [7]. This finding is also supported in vitro by Linfield et al. [8].

Looking over the theoretical perspective, the skin capillaries are the primary site for bilirubin isomerization [12] [13], favored by the current study as well. It is observed clinically that skin discoloration of jaundiced neonates reduced during initial hours of phototherapy, this gives a clue that the isomerization of Z-Z-bilirubin to photobilirubin mainly occurs extravascularly in skin. Due to this finding the neonates were changed from prone to supine position during phototherapy [14]. Few of the studies are contradictory to this point and reported that the efficacy of phototherapy is independent of neonate's position because of underlying intravascular mechanism and this can be confirm by measuring photobilirubin in plasma, 15 minutes after the initiation of phototherapy [12] [15] [16] [17]. This finding is supported by the current study as inverse association of hemoglobin with efficacy of phototherapy could be due to intravascular isomerization of bilirubin.

The hemoglobin influences the efficacy of phototherapy by absorbing the light so its effect on degrading hyperbilirubinemia is not accomplished [18]. Mreihil et al. gave intensive phototherapy to 36 patients and measured hemoglobin, $4 \mathrm{Z}$, and $15 \mathrm{E}$ photoisomers. By doing post-hoc analysis he confirmed a negative association of hemoglobin with Z-E-bilirubin percentage after 15, 30 and 60 minutes but this association disappeared later on. He further explained that after $60 \mathrm{mi}-$ nutes, an equilibrium generated between Z-Z-bilirubin and Z-E-bilirubin in plasma which was not affected by hemoglobin concentration [13]. However, 
current study reported inverse correlation between hemoglobin and bilirubin after 24 hours of phototherapy this could be due to the fact that E-Z-lumirubin is the important isomer for lowering bilirubin while there is no existence of equlibirum theory between Z-Z-bilirubin and Z-E-bilirubin, as favored by few other studies [6] [18] [19].

From a clinical point of view, the current study is important for the physician in treating neonates with hyperbilirubinemia by using phototherapy. Neonates having high hemoglobin concentration need phototherapy for longer duration, with higher light irradiance and larger exposure of body surface-area as compared to neonates with lower concentration of hemoglobin. The main limitation of the current study is that high hemoglobin produces more bilirubin which could show a negative correlation between hemoglobin and $\Delta \mathrm{TsB}_{0-24}$. The strengths of the current studies are that the study participants were homogenous and healthy except the hyperbilirubinemia and all the instruments including hematology analyzer and clinical chemistry analyzer were calibrated before performing test.

\section{Conclusion}

Current study found a significant effect of hemoglobin on efficacy of phototherapy, as the change in total serum bilirubin concentration was inversely correlated with the level hemoglobin. This is important for the physician in treating neonates with hyperbilirubinemia by using phototherapy.

\section{Conflicts of Interest}

The authors declare no conflicts of interest regarding the publication of this paper.

\section{References}

[1] Donneborg, M.L., Vandborg, P.K., Hansen, B.M., Rodrigo-Domingo, M. and Ebbesen, F. (2017) The Impact of Hemoglobin on the Efficacy of Phototherapy in Hyperbilirubinemic Infants. Pediatric Research, 82, 947. https://doi.org/10.1038/pr.2017.186

[2] Maisels, M. (2015) Sister Jean Ward, Phototherapy, and Jaundice: A Unique Human and Photochemical Interaction. Journal of Perinatology, 35, 671. https://doi.org/10.1038/jp.2015.56

[3] Ebbesen, F., Hansen, T.W. and Maisels, M.J. (2017) Update on Phototherapy in Jaundiced Neonates. Current Pediatric Reviews, 13, 176-180. https://doi.org/10.2174/1573396313666170718150056

[4] Ebbesen, F., Madsen, P.H., Vandborg, P.K., Jakobsen, L.H., Trydal, T. and Vreman, H.J. (2016) Bilirubin Isomer Distribution in Jaundiced Neonates during Phototherapy with LED Light Centered at $497 \mathrm{~nm}$ (Turquoise) vs. $459 \mathrm{~nm}$ (Blue). Pediatric Research, 80, 511. https://doi.org/10.1038/pr.2016.115

[5] Mostovnikov, V.A., Mostovnikova, G.R. and Plavski, V.Y. (1995) Spectral and Photochemical Parameters Which Define the Higher Efficacy of Laser Phototherapy in Hyperbilirubinemia in Newborns. 5 th International Conference on Laser Applica- 
tions in Life Sciences, Vol. 2370, 558-561. https://doi.org/10.1117/12.197489

[6] Onishi, S., Isobe, K., Itoh, S., Manabe, M., Sasaki, K., Fukuzaki, R., et al. (1986) Metabolism of Bilirubin and Its Photoisomers in Newborn Infants during Phototherapy. The Journal of Biochemistry, 100, 789-795.

https://doi.org/10.1093/oxfordjournals.jbchem.a121772

[7] Lamola, A.A., Bhutani, V.K., Wong, R.J., Stevenson, D.K. and McDonagh, A.F. (2013) The Effect of Hematocrit on the Efficacy of Phototherapy for Neonatal Jaundice. Pediatric Research, 74, 54. https://doi.org/10.1038/pr.2013.67

[8] Linfield, D.T., Lamola, A.A., Mei, E., Hwang, A.Y., Vreman, H.J., Wong, R.J., et al. (2016) The Effect of Hematocrit on in Vitro Bilirubin Photoalteration. Pediatric Research, 79, 387. https://doi.org/10.1038/pr.2015.240

[9] Curran, S., McMurdy, J.W., Carr, S.R., Muratore, C.S., O’Brien, B.M., Crawford, G.P., et al. (2010) Reflectance Spectrometry for Real-Time Hemoglobin Determination of Placental Vessels during Endoscopic Laser Surgery for Twin-to-Twin Transfusion Syndrome. Journal of Pediatric Surgery, 45, 59-64. https://doi.org/10.1016/j.jpedsurg.2009.10.009

[10] Sikurova, L., Balis, P. and Zvarik, M. (2011) Penetration of Laser Light through Red Blood Cell Ghosts. Journal of Photochemistry and Photobiology B: Biology, 103, 230-233. https://doi.org/10.1016/j.jphotobiol.2011.03.015

[11] Granati, B., Felice, M., Fortunato, A., Giancola, G. and Rubaltelli, F.F. (1983) Sites of Action of Light during Phototherapy. Neonatology, 43, 1-8. https://doi.org/10.1159/000241630

[12] Donneborg, M., Knudsen, K.B. and Ebbesen, F. (2010) Effect of Infants' Position on Serum Bilirubin Level during Conventional Phototherapy. Acta Paediatrica, 99, 1131-1134. https://doi.org/10.1111/j.1651-2227.2010.01885.x

[13] Mreihil, K., Madsen, P., Nakstad, B., Benth, J.Š., Ebbesen, F. and Hansen, T.W.R. (2015) Early Formation of Bilirubin Isomers during Phototherapy for Neonatal Jaundice: Effects of Single vs. Double Fluorescent Lamps vs. Photodiodes. Pediatric Research, 78, 56. https://doi.org/10.1038/pr.2015.61

[14] Hansen, T.W.R. (1996) Therapeutic Approaches to Neonatal Jaundice: An International Survey. Clinical Pediatrics, 35, 309-316. https://doi.org/10.1177/000992289603500604

[15] Bhethanabhotla, S., Thukral, A., Sankar, M., Agarwal, R., Paul, V. and Deorari, A. (2013) Effect of Position of Infant during Phototherapy in Management of Hyperbilirubinemia in Late Preterm and Term Neonates: A Randomized Controlled Trial. Journal of Perinatology, 33, 795. https://doi.org/10.1038/jp.2013.54

[16] Chen, C.M., Liu, S.H., Lai, C.-C., Hwang, C.C. and Hsu, H.-H. (2002) Changing Position Does Not Improve the Efficacy of Conventional Phototherapy. Acta Paediatrica Taiwanica, 43, 255-258.

[17] Mohammadzadeh, A., Bostani, Z., Jafarnejad, F. and Mazloom, R. (2004) Supine versus Turning Position on Bilirubin Level during Phototherapy in Healthy Term Jaundiced Neonates. Saudi Medical Journal, 25, 2051-2052.

[18] Lamola, A.A. (2016) A Pharmacologic View of Phototherapy. Clinics in Perinatolo$g y, 43,259-276$. https://doi.org/10.1016/j.clp.2016.01.004

[19] Okada, H., Masuya, K., Yasuda, S., Okubo, K., Kawada, K., Kusaka, T., et al. (2005) Developmental Changes in Serum Half-Life of (EZ)-Cyclobilirubin. Early Human Development, 81, 619-622. https://doi.org/10.1016/j.earlhumdev.2005.03.014 